Onkologe $2016 \cdot 22: 448-450$

DOI 10.1007/s00761-016-0072-y

(c) Springer-Verlag Berlin Heidelberg 2016

CrossMark

\author{
J. Pratschke $\cdot$ P. M. Schlag ${ }^{2}$ \\ ${ }^{1}$ Chirurgische Klinik, Campus Charité Mitte und Campus Virchow-Klinikum, Charité - Universitätsmedizin \\ Berlin, Berlin, Deutschland \\ ${ }^{2}$ Charité Comprehensive Cancer Center, Campus Charité Mitte, Charité - Universitätsmedizin Berlin, Berlin, \\ Deutschland
}

\title{
Transplantationen in der Onkologie
}

\author{
Aktuelle Standards und \\ Zukunftsperspektiven
}

Die Behandlungsergebnisse transplantierter Patienten wurden in den letzten Dekaden maßgeblich durch folgende drei Faktoren kontinuierlich verbessert:

- Interdisziplinäre Patientenselektion vor geplanter Transplantation.

- Multiprofessionelle Nachbetreuung nach stattgehabter Transplantation.

- Gezielter und steuerbarer Einsatz von Immunsuppressiva.

So konnte die Transplantation von autologen Zellen oder ganzen Organen sowohl für Patienten, welche an einem Organversagen leiden, als auch für Patienten mit bestimmten malignen Grunderkrankungen als sicheres Therapieverfahren etabliert werden. Da die Indikationen zur Transplantation auf dieser Grundlage immer weiter ausgedehnt wurden, gibt es in manchen Bereichen (z. B. bei Leber- oder Nierentransplantationen) bereits Organengpässe, da es in Deutschland weit mehr Patienten auf Wartelisten zur Transplantation als verfügbare Organe gibt.

Ziel der vorliegenden Abhandlung ist die Darstellung der aktuellen Standards von etablierten Transplantationsverfahren bei onkologischen Patienten sowie die Vorstellung von neuartigen Verfahren und deren Perspektiven.

J. Arend et al. resümieren in ihrem Beitrag, dass die Lebertransplantation (LTx) mittlerweile ein gut etabliertes Verfahren für Patienten mit einem hepatozellulären
Karzinom (HCC) bei Leberzirrhose ist. In diesen Fällen wird sowohl der Tumor als auch die Grunderkrankung kurativ therapiert. Unter Einbeziehung der sog. Milan-Kriterien können inzwischen 5-Jahres-Überlebensraten von $75 \%$ für adulte Patienten erreicht werden. Hierbei handelt es sich um Kriterien zur Abschätzung der Erfolgsaussichten bei Lebertransplantationen. Bei Kindern steht diesbezüglich das Hepatoblastom mit einer 5-Jahres-Überlebensrate von ebenfalls $75 \%$ an erster Stelle. Lebertransplantationen bei anderen Erkrankungen, z. B. dem Cholangiokarzinom oder bei Lebermetastasen, werden im Rahmen von weiteren Studien evaluiert. Mit einer großzügigen weiteren Ausweitung der Indikationen zur LTx ist aber aufgrund des Organmangels derzeit eher nicht zu rechnen.

Langzeitüberlebensdaten transplantierter Patienten zeigen leider eindeutig, dass diese Patienten ein deutlich erhöhtes Risiko haben, De-novo-Malignome zu entwickeln. J. Mittler und H. Lang erörtern, dass ursächlich hierfür, neben individuellen Risikofaktoren, vor allem die Immunmodulation nach Transplantation anzusehen ist. Zusätzlich treten bei bestimmten Tumorentitäten durch die Immunmodulation häufig onkogene virale Infektionen auf. Neben den üblichen onkologischen Vorsorge- und Behandlungsmaßnahmen kommen dem engmaschigen Tumorscreening und der antiviralen Prophylaxe immer mehr Bedeutung zu. Auch die Vermeidung bestimmter immunsupprimierender Faktoren, vor dem Hintergrund des individuellen Risikoprofils, ist für das Outcome sehr wichtig.

Die hämatopoetische Stammzelltransplantation (HSZT) stellt eine kurative Therapieoption für viele hämatoonkologische Erkrankungen dar. F. Ayuk und N. Kröger geben einen Überblick, wie die Verbesserungen der Ergebnisse in den letzten Jahrzehnten Schritt für Schritt erreicht werden konnten, und wie neue Entwicklungen in der Stammzellmobilisierung und -therapie inzwischen auch zu Erweiterungen der Indikation geführt haben. Durch Minimierung der therapiebedingten Nebenwirkungen und der Mortalität kann die Stammzelltransplantation als Therapieoption nunmehr auch bei älteren Patienten erfolgreich angewandt werden. Moderne Therapiekonzepte schließen die HSZT nicht nur als Ultima Ratio, sondern als Teil eines therapeutischen Konzepts mit entsprechenden Prä- und Posttransplantationstherapien ein, um die Heilungschancen der Patienten mit hämatoonkologischen Erkrankungen stetig zu verbessern.

B. Strücker et al. legen in einem sehr interessanten Artikel dar, wie auch bei Patienten mit soliden Tumoren (z. B. mit primären oder sekundären Malignomen der Leber) die Transplantation von hämatopoetischen Stammzellen oder mesen- 
Hier steht eine Anzeige.

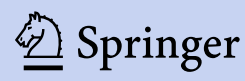


chymalen Stromazellen zunehmend an Stellenwert gewinnen könnte. Mit diesem Vorgehen kann ein positiver Effekt auf die Leberregeneration ausgelöst werden. Somit können diese Zellen zur Augmentation der Leber vor oder nach onkologischer Resektion genutzt werden. Erste klinische Ergebnisse aus Heilversuchen und das Tiermodell zeigten, dass parakrine Effekte der Stammzellen und der horizontale Transfer von MicroRNA, die die Zell-Zell-Kommunikation beeinflusst, für eine verbesserte Leberregeneration bedeutsam sind. Inwieweit diese Verfahren zukünftig Konsequenzen für die klinische Versorgung von Patienten mit Malignomen der Leber haben, wird derzeit durch Studien weiter geklärt.

Ferner wird die Option einer autologen Inseltransplantation bei chirurgisch indizierten Resektionen des Pankreas behandelt, da auf diesem Gebiet erhebliche Fortschritte erzielt wurden. Die bislang vorliegenden Daten aus internationalen Zentren zeigen, dass diese Therapieform auch bei neoplastischen Pankreasveränderungen sicher und effizient durchgeführt werden kann. Aus diesem Grund wird zukünftig die Indikation zur Inselautotransplantation sicherlich ausgedehnt.

Unter radioonkologischen Gesichtspunkten hat sich in der Planung und Durchführung bei der Stammzelltransplantation relativ wenig verändert, weshalb auf eine diesbezügliche Wiederholung der Kriterien verzichtet wurde.

Ein wichtiger und derzeit unterrepräsentierter Bereich in der Transplantationsmedizin ist die psychologische Situation und Befindlichkeit der Patienten nach Transplantationen. Diesen Aspekten, und dabei vor allem dem Suizidrisiko nach HSZT, widmet sich das systematische Review der Datenlage von J. Hefner und H. Csef. Es wird darin aufgezeigt, dass durch die spezielle Situation einer HSCT spezifische Kommunikationsstörungen auftreten können, die, unabhängig von depressiven Verstimmungen, das Suizidrisiko erhöhen.

Wir danken allen Autoren für ihre kompetenten und aktuellen Beiträge, die sicher auf großes Interesse bei unseren Lesern stoßen werden. Zeigen sie doch, dass die Transplantation auch in der Tu- mormedizin eine wichtige, und nicht nur in der Hämatoonkologie, kurative Behandlungsoption sein kann, deren Weiterentwicklung und Perspektiven zu berechtigten Hoffnungen Anlass geben.

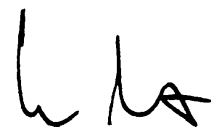

Prof. Dr. J. Pratschke

für die Schriftleiter

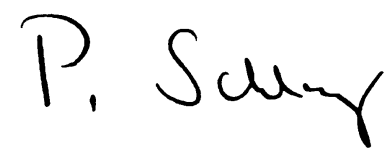

Prof. Dr. P. M. Schlag

für die Herausgeber

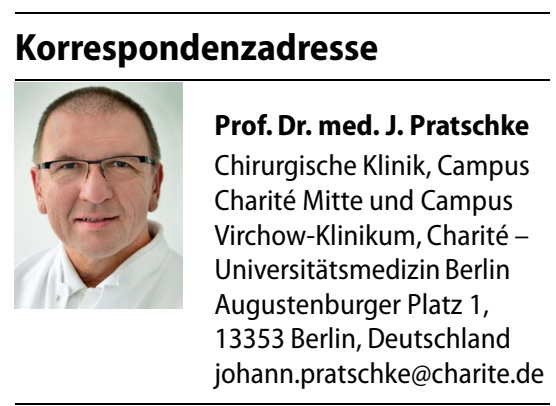

Interessenkonflikt. J. Pratschke und P.M. Schlag geben an, dass kein Interessenkonflikt besteht.

\section{ONKO-Internetportal}

Aktuelles von der Jahrestagung der EHA

Aktuelles und Informatives von den onkologischen Sommerkongressen 2016 bie-

tet das ONKO-Internetportal in Kooperation mit der Deutschen Krebsgesellschaft in seiner Rubrik Kongressberichte. Derzeit im Fokus: die Jahrestagung der European Hematology Association (EHA) und neueste Erkenntnisse zu bösartigen Erkrankungen des blutbildenden Systems.

Steigende Teilnehmerzahlen und eine starke Resonanz auch im außereuropäischen Raum belegen die zunehmende Bedeutung des EHA-Kongresses, der diesmal in Kopenhagen stattfand. Hämatologen aus ganz Europa diskutierten dort auf Basis eines umfangreichen wissenschaftlichen Programms die Bedeutung aktueller Forschung für den klinischen Alltag. Vor Ort sprach das Redaktionsteam des ONKO-Internetportals mit Experten, die in Interviews und Gesprächsrunden eine zeitnahe Einordnung der präsentierten Studiendaten vornahmen.

Zu den besonderen Highlights, die Ärzte ab sofort online abrufen können, zählt das State-of-the-Art-Gespräch zum multiplen Myelom, das führende deutsche Experten der Hämatoonkologie versammelt. Alle Interviews, Expertengespräche und Kongressberichte sind einsehbar unter www.krebsgesellschaft.de/eha2016.

Quelle

Deutsche Krebsgesellschaft e.V. (DKG) www.krebsgesellschaft.de 